\title{
Erratum: Temperature dependence of nuclear fission time in heavy-ion fusion-fission reactions [Phys. Rev. C 96, 054611 (2017)]
}

\author{
Chris Eccles, Sanil Roy, Thomas H. Gray, and Alessio Zaccone
}

(Received 25 January 2018; published 12 July 2018)

DOI: 10.1103/PhysRevC.98.019905

We wish to make the following corrections to the original article.

Equation (7) of the original article contains a typographical misprint. It should read

$$
\tau_{f}=\frac{\eta}{T} L^{2}\left[\frac{1}{2}+\frac{1}{6}\left(\frac{U_{f}}{T}\right)+\text { h.o.t. }\right] .
$$

All results of the article remain unaffected.

We also note that in the original paper to the fission time data of Ref. [1] was referred to as "experimental data". To be more precise, those fission lifetime data are not "first-hand" experimental data but the result of statistical model calculations with the CASCADE code, which have been adjusted based on experimental fragment, neutron, and $\gamma$ multiplicity data. In particular, the symbols in Fig. 4 of our article were extracted from Fig. 6 of Ref. [1] and were corrected in the following way. The data in Fig. 6 of Ref. [1] account for both the time to reach the saddle and to include the saddle-to-scission time. Since our improved fission lifetime formula Eq. (6) of the original paper provides the fission lifetime defined as the time to reach the saddle point from the bottom of the well of the compound nucleus (hence without the saddle-to-scission time), the data of Ref. [1] had to be corrected. Hence before plotting them in Fig. 4 of the original paper, we subtracted off the saddle-to-scission time using Eq. (10) of Ref. [1] which provides an estimate of the saddle-to-scission time for the system under consideration with the parameters suggested in Ref. [1] as appropriate for the system under consideration. This correction ensures the consistency of the plotted quantity (the fission lifetime measured up to the saddle) between our formula and the data of Ref. [1].

We gratefully acknowledge input and feedback from I. I. Gontchar and M. V. Chushnyakova, who brought these issues to our attention.

[1] I. Diószegi, N. P. Shaw, I. Mazumdar, A. Hatzikoutelis, and P. Paul, Phys. Rev. C 61, 024613 (2000). 